\title{
BIOSORCIÓN DEL COLORANTE AZUL DE METILENO USANDO LOS CLADODIOS DE LA TUNA (Opuntia ficus indica)
}

\author{
Carmencita Lavado Meza ${ }^{* a}$, Rosario Sun Kou ${ }^{\mathrm{b}}$, Tracy Castro Arroyo ${ }^{\mathrm{c}}$
}

\begin{abstract}
RESUMEN
La biosorción es un proceso efectivo para la remoción de colorantes sintéticos desde soluciones acuosas. Se estudió el uso de la biomasa de los cladodios de la tuna (BCT) para remover el colorante catiónico azul de metileno (AM) de la solución acuosa. Las técnicas de caracterización: FTIR, SEM y punto de carga cero (pHPCC) muestran las características químicas y físicas de la BCT. Se evaluaron los parámetros experimentales que afectan el proceso de biosorción, como el $\mathrm{pH}$, tiempo de contacto y la dosis de biomasa/volumen, en un sistema estacionario. Los datos experimentales del equilibrio se ajustaron al modelo de la isoterma de Langmuir, la capacidad máxima de biosorción del AM fue de 156,25 mg/g con una dosis de BCT/volumen de $4 \mathrm{~g} / \mathrm{L}$, pH 8 y tiempo de contacto de $120 \mathrm{~min}$. Los datos cinéticos se pueden describir bien con el modelo de pseudo-segundo orden, la biosorción parece estar controlada por la quimisorción y puede estar involucrada con la adsorción sobre la superficie y la difusión en los poros durante todo el proceso de biosorción. Se demostró que la $\mathrm{BCT}$ se constituye como un biomaterial prometedor, eficiente y biodegradable para eliminar el AM de las aguas residuales.
\end{abstract}

Palabras clave: biosorción, isotermas, azul de metileno, cinética.

\section{BIOSORPTION OF DYE METHYLENE BLUE USING THE CLADODES OF THE TUNA (Opuntia ficus indica)}

\begin{abstract}
Biosorption is an effective process for removing synthetic dyes from aqueous solutions. The use of prickly pear cladode biomass (PCB) was studied to remove the methylene blue cationic dye (MB) from the aqueous solution. The characterization techniques: FTIR, SEM and point of zero load (pHPZC) show the chemical and physical characteristics of PCB. Experimental parameters that affect the biosorption process, such as $\mathrm{pH}$, contact time and

\footnotetext{
a Facultad de Ingeniería Ambiental, Universidad Continental Av. San Carlos 1980, Huancayo, Perú, carmencita. lavado@pucp.pe

b Sección Química. Departamento Académico de Ciencias, Pontificia Universidad Católica del Perú, Av. Universitaria 1801, Lima, Perú.

c Facultad de Ingeniería Ambiental, Universidad Alas Peruanas, Coronel Parra 2036, Huancayo, Perú.
} 
biomass / volume dose, were evaluated in a continuous system. The equilibrium experimental data were adjusted to the Langmuir isotherm model, the maximum biosorption capacity of the MB was $156.25 \mathrm{mg} / \mathrm{g}$ with a dose of PCB/volume of $4 \mathrm{~g} / \mathrm{L}, \mathrm{pH} 8$ and contact time of $120 \mathrm{~min}$. Kinetic data can be well described with the pseudo-second order model, biosorption appears to be controlled by chemisorption and may be involved with adsorption on the surface and diffusion into the pores throughout the biosorption process. PCB was shown to be a promising, efficient, and biodegradable biomaterial for removing AM from wastewater.

Key words: Biosorption, isotherms, methylene blue, kinetics.

\section{INTRODUCCIÓN}

Los colorantes sintéticos se usan ampliamente en todo el mundo en una amplia gama de productos de las industrias textiles, papeleras, alimentarias, cosméticas y farmacéuticas ${ }^{1}$, estos colorantes, son estables y no biodegradables y están diseñados para mantener el color en varios materiales y ser resistentes al agua, jabón y agentes oxidantes ${ }^{2}$. Las aguas residuales que contienen colorantes al ser descargadas en los recursos hídricos inhiben la penetración de la luz solar y disminuyen la acción fotosintética, así mismo estas aguas se caracterizan por poseer una alta demanda química de oxígeno (DQO), $\mathrm{pH}$ alto y toxicidad biológica $^{3}$. La mayoría de los colorantes sintéticos son tóxicos y cancerígenos por lo que, si las aguas residuales que contienen estas sustancias no se tratan de manera efectiva, podrían ser responsables de daños ambientales graves y también representar una amenaza para la salud pública ${ }^{4}$. Los colorantes sintéticos se pueden clasificar como aniónicos, catiónicos y no iónicos. El azul de metileno (AM), clasificado en la categoría de colorante catiónico, es uno de los tintes ampliamente usados en la industria para teñir algodón, madera y seda ${ }^{5}$. El AM es tóxico, si se ingieren en grandes cantidades, produce efectos nocivos como gastritis, dolor de cabeza intenso, micción dolorosa y síndromes similares a la metahemoglobinemia, sudoración profusa, confusión mental y toxicidad respiratoria ${ }^{6}$. Por lo general, las aguas residuales coloreadas se tratan mediante procesos fisicoquímicos como ultrafiltración, coagulación, fotooxidación, pero estos tratamientos tienen limitaciones como: formación de subproductos tóxicos, para su funcionamiento requieren grandes cantidades de energía y no se adaptan a la amplia gama de tintes que existen en el mercado ${ }^{7}$, las desventajas de estos métodos han motivado a investigar métodos alternativos para la eliminación de colorantes, una de ellas es la adsorción, esta técnica se ha empleado ampliamente para la eliminación de colorantes donde se utiliza un adsorbente sólido para atraer la molécula de colorante y finalmente conducir a su eliminación del medio acuoso, el material más usado como adsorbente es el carbón activado, pero su uso es limitado debido a su alto costo y baja capacidad de regeneración, estas limitaciones llevaron a los investigadores a explorar la adsorción o biosorción usando materiales alternativos abundantes, disponibles y de bajo costo, como subproductos agrícolas (hojas, grano, semillas, raíz, etc.) hongos, plantas, algas y bacterias ${ }^{2}$. La tuna (Opuntia ficus indica) es una especie de naturaleza xerófila, de la familia de los cactáceas es nativa de México pero se naturalizó en toda la cuenca mediterránea y en las zonas templadas de América, África, Asia y Oceanía, que se encuentra en abundancia en 
la región sierra en Perú ${ }^{8}$. Los cladodios del cactus están constituidos principalmente por un heteropolisacárido con un peso molecular de 23104 a $3106 \mathrm{~g} / \mathrm{mol}$, el mucílago es una mezcla de polisacáridos ácidos y neutros que consisten principalmente en arabinosa; galactosa ácido galacturónico; ramnosa y xilosa, los grupos funcionales de estos compuestos podrían actuar con las moléculas de los colorantes removiéndolos de la solución matriz ${ }^{9}$. El objetivo del presente estudio fue evaluar la potencialidad de la biomasa de los cladodios de la tuna (BCT) para ser usado como biosorbente para remover el colorante azul de metileno de una solución acuosa, constituyéndose así como una alternativa eficiente para eliminar colorantes de las aguas residuales, para ello se caracterizó física y químicamente la BCT y se evaluó la influencia de la dosis de la biomasa en un volumen fijo de la solución con AM (g/L), pH y tiempo, sobre la capacidad de remoción del AM.

\section{PARTE EXPERIMENTAL}

\section{Preparación del biosorbente:}

Los cladodios del cactus fueron recolectados en el distrito de Pilcomayo Región Junín, se eliminaron los residuos con abundante agua destilada, se retiraron las espinas y se cortaron en trozos pequeños y posteriormente se llevaron a secar al horno a $60^{\circ} \mathrm{C}$ durante 72 horas, el material seco fue molido y tamizado a malla $70 \mu \mathrm{m}$, obteniéndose de esta manera la biomasa de los cladodios de la tuna (BCT).

\section{Caracterización del biosorbente:}

Los grupos funcionales presentes en la superficie de biosorbente se identificaron por espectroscopia infrarroja con transformadas de Fourier (FTIR) en un Espectrofotómetro SHIMADZU FTIR- 8700 la muestra se analizó en un rango espectral de 4000 a $400 \mathrm{~cm}^{-1}$. La microscopía electrónica de barrido (SEM) modelo SU 8230Evo15, marca Hitachi, se utilizó para estudiar la morfología de la superficie del adsorbente. La determinación de los grupos ácidos se llevó a cabo siguiendo el método Boehm, descrito por Aygün et al. ${ }^{10}$ en el que se emplea una base fuerte, como el $\mathrm{NaOH}$, para neutralizar los centros ácidos presentes en la superficie de la BCT, se pesó 0,25 de la BCT, se agregó $50 \mathrm{~mL}$ de $\mathrm{NaOH} \mathrm{0,05} \mathrm{M} \mathrm{(estandarizado}$ con biftalato ácido de potasio) esta mezcla se agitó por 24 horas a una velocidad de $100 \mathrm{rpm}$, luego se tomó $20 \mathrm{~mL}$ de la solución remanente y se tituló con $0,05 \mathrm{M}$ de $\mathrm{HCl}$ (estandarizado con la solución $0,05 \mathrm{M}$ de $\mathrm{KOH}$ ) usando como indicador azul de bromocresol. El punto final es indicado por el viraje de azul a verde de la solución, con el gasto obtenido (mL) se calculó los moles de grupos ácidos, los resultados se expresaron en mmolH+/g BCT. Para la determinación del pHPCC se procedió de acuerdo a lo reportado por Moghazy, et al. ${ }^{5}$ Para ello se prepararon soluciones con diferentes $\mathrm{pH}$, mezclando en cada solución $50 \mathrm{~mL}$ de agua destilada con $0,1 \mathrm{~g}$ de $\mathrm{BCT}$ en un matraz erlenmeyer con tapa, el $\mathrm{pH}$ se varió con soluciones de $\mathrm{NaOH} 0,1 \mathrm{M}$ y $\mathrm{HCl} 0,1 \mathrm{M}$, la mezcla se mantuvo en agitación constante (150 rpm) durante $15 \mathrm{~min}$, trascurrido este tiempo se midió el $\mathrm{pH}$ final ( $\mathrm{pHf}$ ), todos los datos se obtuvieron por triplicado. Se graficaron los valores de $\Delta \mathrm{pH}\left(\Delta \mathrm{pH}=\mathrm{pH}_{\mathrm{o}}-\mathrm{pH}_{\mathrm{f}}\right)$ versus $\mathrm{pH}$ inicial (pHo), el punto de carga cero ( $\mathrm{pHPCC}$ ) se obtuvo de la gráfica cuando $\Delta \mathrm{pH}=0$. 


\section{Biosorción del azul de metileno:}

Se preparó una solución stock de AM de $500 \mathrm{mg} / \mathrm{L}$ a partir de ella se prepararon las diferentes concentraciones necesarias, la biosorción del AM se llevó a cabo en un sistema discontinuo, utilizando $50 \mathrm{~mL}$ de muestra de solución del colorante en matraces Erlenmeyer en un equipo de agitación múltiple marca Velp, a una velocidad 150 RPM y temperatura $20^{\circ} \mathrm{C}$, se realizó el estudio de la influencia de la dosis usando diferentes cantidades de BCT en un rango de 0,025 - 0,3 g. Para determinar el equilibrio cinético, se ensayó a diferentes intervalos de tiempo (5 -180 min). El equilibrio de biosorción se evaluó a diferentes concentraciones iniciales de AM (20-300 mg/L de AM). El estudio de la influencia del pH se realizó ajustando el $\mathrm{pH}$ a valores comprendidos entre 2,0 - 8,0 con soluciones de $\mathrm{HCl}$ y $\mathrm{NaOH}$ 0,1 $\mathrm{M}$. Todos los experimentos se llevaron a cabo por duplicado y los resultados presentados son las medias correspondientes. En cada uno de los casos luego del experimento, las fases se separaron por filtración. Las concentraciones de colorante AM en las muestras se midieron mediante un espectrofotómetro UV (Beckman Du 64 UV/VIS) a una longitud de onda de $663 \mathrm{~nm}$. La cantidad de AM retenido por el biosorbente (qe) y el porcentaje de biosorción se calcularon mediante las ecuaciones 01 y 02 , respectivamente.

$$
\begin{aligned}
& q_{e}=\frac{\left(C_{0}-C_{e}\right)}{M} x V \\
& \% R=\frac{\left(C_{0}-C_{e}\right)}{C_{0}} \times 100
\end{aligned}
$$

Donde: Co y Ce son la concentración inicial y final del AM en la solución (mg/L) antes y después de la biosorción respectivamente, $\mathrm{M}$ es la masa del $\mathrm{BCT}(\mathrm{g})$, y $\mathrm{V}$ es el volumen de la solución (L). 


\section{RESULTADOS Y DISCUSIÓN}

Caracterización de la biomasa:

Para comprender cualquier posible interacción entre los grupos funcionales de la superficie de la BCT y el colorante MB, se estudió la BCT utilizando espectroscopía FTIR (figura 1)

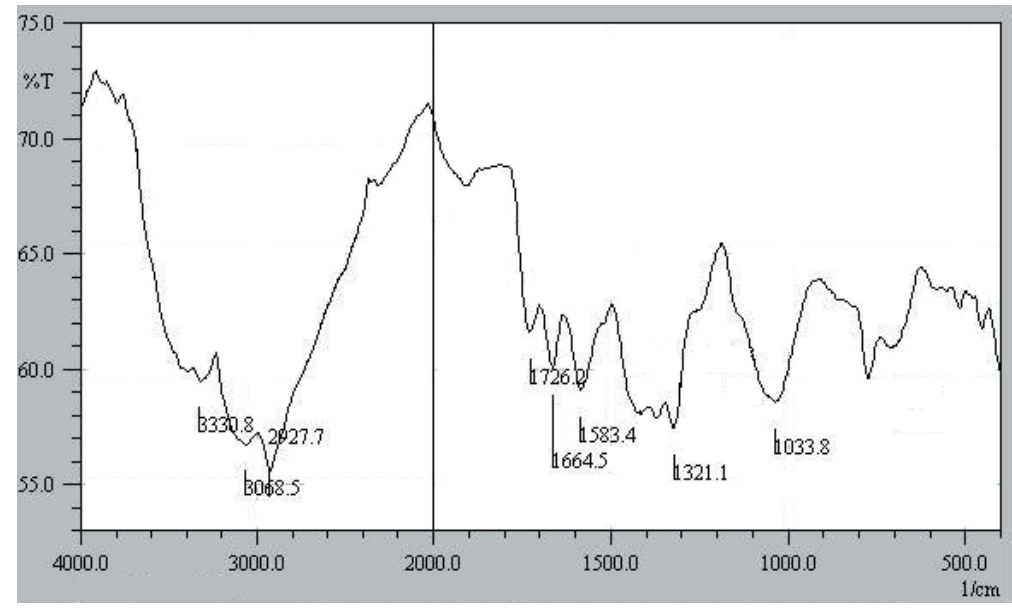

Figura 1. Espectro FTIR de la biomasa de la BCT.

El análisis espectroscópico evidenció una banda ancha en el rango espectral de 3600-3000 cm-1 que se le atribuye a las vibraciones de estiramiento del $\mathrm{O}-\mathrm{H}$ y $\mathrm{N}-\mathrm{H}$, lo que indica la presencia del $-\mathrm{OH}$ en carboxilos y presencia de aminas ${ }^{2}$, otra banda a $2927 \mathrm{~cm}^{-1}$ debido a la vibración asimétrica del enlace $\mathrm{C}-\mathrm{H}$ de los grupos alifáticos, la banda a $1726 \mathrm{~cm}-1$ se le atribuye a la deformación del $-\mathrm{C}=\mathrm{O}$, la banda a $1664,5 \mathrm{~cm}-1$ es debido a las vibraciones del enlace $\mathrm{C}=\mathrm{C}$ aromático ${ }^{11}$. La banda ancha a $1033 \mathrm{~cm}^{-1}$ se atribuye a la deformación simétrica de alquil éteres y C-O-C presentes en los polisacáridos ${ }^{12}$. Los picos a 1583,4 y $1321,1 \mathrm{~cm}^{-1}$ corresponden al estiramiento y deformación, respectivamente del enlace $\mathrm{C}-\mathrm{N}$ presentes en aminas aromáticas ${ }^{13}$. La presencia de grupos hidroxilo $\mathrm{OH}$, carbonilo $\mathrm{C}=\mathrm{O}$, éteres $\mathrm{C}-\mathrm{O}-\mathrm{C}$ en grupos aromáticos, aminas aromáticas, en la estructura lignocelulósica del biosorbente le confiere a la BCT capacidad para interactuar con el AM.

Se determinó la cantidad de grupos ácidos mediante el método Boehm, el cual demostró que la BCT posee una acidez total superficial de $7,34 \mathrm{mmolH}+/ \mathrm{g}$, que puede deberse a la presencia de los grupos funcionales reportados en el análisis del espectro FTIR (figura 1), los cuales podrían interactuar con el colorante catiónico AM. 
La morfología de la superficie del biosorbente se caracterizó por SEM. Como se ve en la figura 2, el biosorbente exhibe una morfología de superficie heterogénea y porosa (figura 2A), tal forma estructural facilitaría el proceso de biosorción porque proporciona un área superficial. Por otro lado, después de la biosorción se observa una morfología superficial más homogénea y con menos presencia de cavidades. Esto se debería a la ocupación de la superficie biosorbente por las moléculas de colorante ${ }^{2}$.
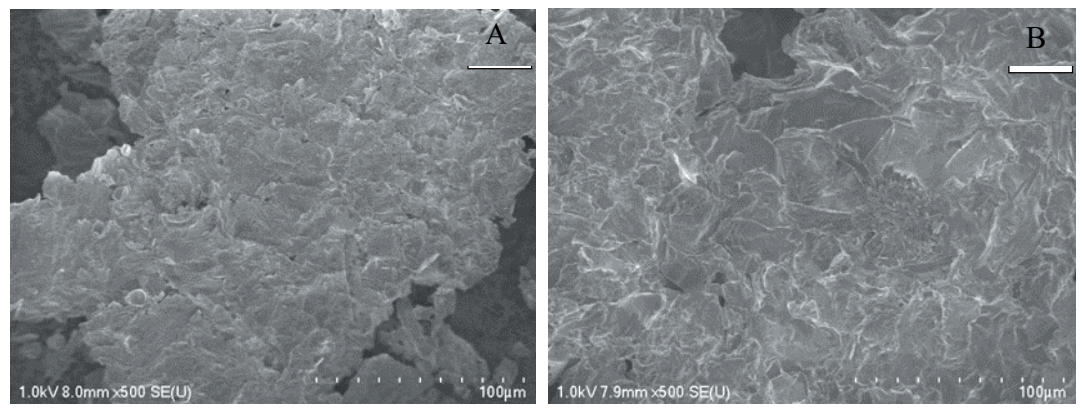

Figura 2. SEM de la BCT A) Antes de la biosorción B) Después de la biosorción.

El pHPCC es el pH al cual la carga superficial neta en el biosorbente es cero, muestra la tendencia de la superficie biosorbente para cargarse positiva o negativamente dependiendo del $\mathrm{pH}$, si el $\mathrm{pH}<\mathrm{pHPCC}$ La superficie del biosorbente está cargada positivamente, favoreciéndose la biosorción de colorantes aniónicos, por otro lado, si el pH > pHPCC, la superficie biosorbente está cargada negativamente y esto favorece la biosorción de colorantes catiónicos ${ }^{14}$. La figura 3 muestra que el pHPCC de la BCT es 4,3. Por lo tanto a $\mathrm{pH}>4,3$ la densidad de carga negativa en la superficie del biosorbente aumenta y, por lo tanto, se logra una mayor capacidad de biosorción debido a la creciente atracción electrostática entre las moléculas de colorante catiónico y los grupos funcionales del material biosorbente.

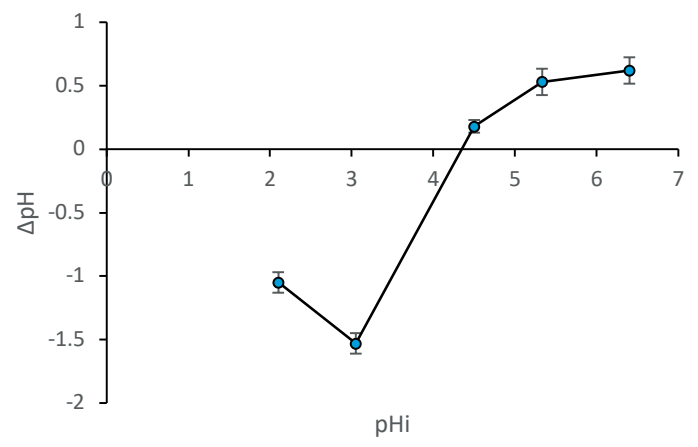

Figura 3. Determinación del pHPCC de la BCT. 


\section{Influencia de la dosis del biosorbente:}

Los datos obtenidos de los experimentos con diferentes dosis de biosorbente en $50 \mathrm{~mL}$ de solución, se presentan en la figura 4. El aumento de la dosis de la BCT de 0,025 a 0,3g da como resultado un incremento en el rendimiento de la biosorción de $51 \%$ a $98 \%$, respectivamente, este comportamiento podría deberse a un aumento en el número de posibles sitios de unión y área de superficie del biosorbente. Un aumento adicional en la concentración de biomasa sobre $0,2 \mathrm{~g} / 50 \mathrm{~mL}$ no condujo a una mejora significativa en el rendimiento de la biosorción, esto podría explicarse como consecuencia de una aglomeración parcial de biomasa, que resulta en una disminución en el área de superficie específica efectiva para la biosorción ${ }^{15}$. Se logró una máxima $\% \mathrm{R}$ con una dosis de BCT con $0,2 \mathrm{~g} / 50 \mathrm{~mL}$ equivalente a $4 \mathrm{~g} / \mathrm{L}$, motivo por lo cual esta dosis se fijó para todos los experimentos siguientes. Esta misma tendencia lo reportan Vijayaraghavan et $a .^{7}{ }^{7}$ quienes removieron AM usando una alga marina, donde alcanzaron una máxima $\% \mathrm{R}$ con una dosis de $5 \mathrm{~g} / \mathrm{L}$.

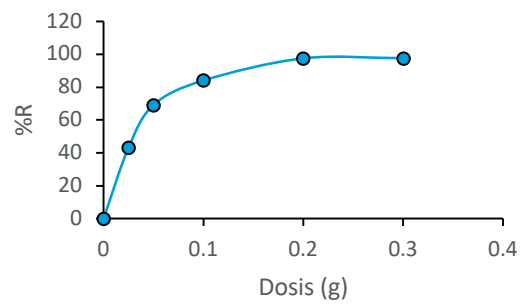

Figura 4. Influencia de la dosis de la BCT sobre el $\% \mathrm{R}, \mathrm{V}=50 \mathrm{~mL}, \mathrm{Ci}=50 \mathrm{mg} / \mathrm{L}$, $\mathrm{T}=20 \mathrm{oC}, \mathrm{t}=120 \mathrm{~min}$.

\section{Influencia del tiempo de contacto y cinética de biosorción:}

Los resultados del estudio de la influencia del tiempo sobre la capacidad de biosorción, se muestran en la figura 5.

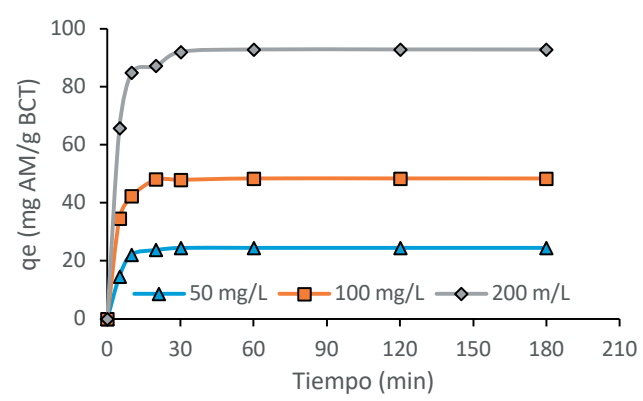

Figura 5. Efecto del tiempo de contacto sobre la biosorción de AM, masa BCT/ volumen $=4 \mathrm{~g} / \mathrm{L}, \mathrm{T}=20 \mathrm{oC}$. 
Se puede observar que para diferentes concentraciones iniciales la capacidad de biosorción aumentó rápidamente en los primeros 10 minutos y luego aumentó lentamente hasta alcanzar el equilibrio a los 120 minutos. La fase rápida inicial puede atribuirse a la existencia de una superficie descubierta de la BCT para el proceso de biosorción, posteriormente, a medida que los sitios de adsorción de la superficie se saturaron, la velocidad de biosorción disminuyó con el tiempo de contacto adicional. Por lo tanto, el tiempo de contacto óptimo se fijó en 120 minutos para el resto de los experimentos. Se observó que la cantidad de AM biosorbido aumentó con un aumento en la concentración inicial del colorante, esto puede explicarse en el supuesto que la fuerza impulsora para superar la resistencia a la transferencia de masa entre la fase sólida y líquida fue mayor en el caso de una mayor concentración de $\mathrm{MB}^{16}$. Varios investigadores informaron resultados similares de resultados para la adsorción de AM sobre residuos de eucalipto ${ }^{4}$, algas $^{5}$, pepas de frutas y cáscaras de nuez ${ }^{10}$.

\section{Estudio cinético:}

Con la finalidad de investigar el mecanismo del proceso de eliminación del colorante AM, los datos cinéticos de biosorción se modelaron utilizando los modelos cinéticos de difusión pseudo-primer orden, pseudo-segundo orden y de difusión intrapartícula, ecuación 3,4 y $6^{14}$ :

$$
\begin{aligned}
& \log \left(q_{e}-q_{t}\right)=\log \left(q_{e}\right)-\frac{k_{1}}{2.303} t \\
& \frac{t}{q_{t}}=\frac{1}{k_{2} q_{e}^{2}}+\frac{t}{q_{e}} \\
& h=k_{2} q_{e}^{2} \\
& q_{t}=k_{i d} t^{1 / 2}+B
\end{aligned}
$$

Donde, qt y qe es la cantidad de ion metálico retenido por unidad de masa de sorbente en un tiempo t y en el equilibrio, respectivamente $(\mathrm{mg} / \mathrm{g}), \mathrm{k} 1$ la constante cinética de primer orden ( $1 / \mathrm{min}), \mathrm{k}_{2}$ (g/mg*min) es la constante de velocidad de adsorción y h es la tasa de adsorción inicial ( $\mathrm{mg} / \mathrm{g} \mathrm{min}), k_{i d}$ es la constante de velocidad de difusión intraparticular $\left(\mathrm{mg} / \mathrm{g} * \mathrm{~min}^{1 / 2}\right)$ y B es una constante relacionada con el espesor de la capa límite del adsorbente $(\mathrm{mg} / \mathrm{g}$ )

Los parámetros cinéticos de la ecuación de pseudo primer orden, pseudo segundo orden se calcularon luego de graficar Log (qe-qt) vs t (figura 6A), t/qt Vs t (figura 6B), y del segundo tramo lineal del gráfico qt vs $\mathrm{t}^{\wedge} 0,5$ (figura $6 \mathrm{C}$ ), respectivamente. Los resultados se presentan en la tabla 1, como se observa, los valores del coeficiente de correlación $\left(\mathrm{R}^{2}\right)$ del modelo de pseudo-segundo orden es igual a 0,999 para todos los valores de $\mathrm{pH}$ y las qe calculadas a partir del modelo de pseudo-segundo orden coinciden muy bien con los datos experimentales, en comparación con el modelo de primer orden. Por lo tanto, la cinética de biosorción está mejor representada por el modelo de pseudo-segundo orden y esta se basa en el supuesto de que el paso de control de la velocidad puede ser quimisorción que involucraría intercambio de electrones entre el AM y los grupos funcionales (principalmente grupos hidroxilo y carboxilo) de la $\mathrm{BCT}^{15}$, como se observa en la tabla 1 , la tasa de adsorción inicial $\left[\mathrm{h}=\mathrm{k} 2\left(\mathrm{q}_{\mathrm{e}}\right)^{2}\right]$ 
aumentó con el incremento de la concentración inicial de colorante, lo que indica que la capacidad de biosorción y la tasa de biosorción fueron más altas en las etapas iniciales. Esto podría estar respaldado por el aumento de la fuerza impulsora para la transferencia de masa a concentraciones iniciales más altas que permite que más moléculas de colorante alcancen la superficie de la BCT en un tiempo más corto ${ }^{16}$. Los modelos cinéticos de pseudo primer orden y pseudo segundo orden no son suficientes para explicar el mecanismo de difusión, por lo tanto, los resultados cinéticos se analizaron adicionalmente mediante el modelo de difusión intrapartícula (figura 6C y 6D) según este modelo, si la regresión de qt versus la raíz cuadrada del tiempo $\left(\mathrm{t}^{1 / 2}\right)$ es lineal, entonces la difusión intrapartícula está involucrada en el proceso de biosorción y si B es cero entonces la difusión intrapartícula es el único paso limitante de la velocidad. Como se muestra en la figura 6C, las gráficas no fueron lineales en todo el rango de tiempo, lo que revela que la difusión de poros no es el paso limitante de la velocidad y que tanto la adsorción superficial como la difusión intrapartícula existirían durante el proceso de biosorción ${ }^{17}$.
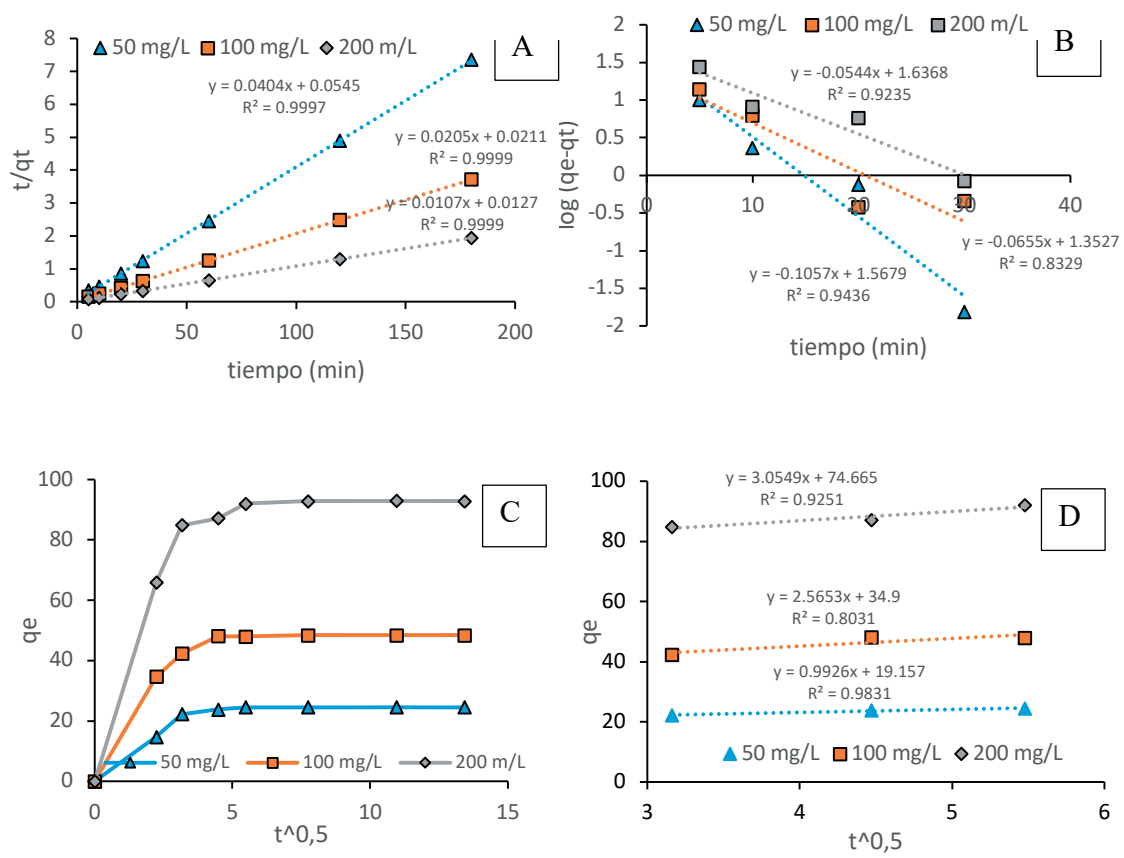

Figura 6. Cinética de biosorción del AM sobre la BCT A) pseudo primer orden B) pseudo segundo orden C) Difusión intrapartícula D) segunda porción lineal qe $\mathrm{Vs}^{\wedge} \mathrm{t}^{\wedge 0,5}$. 
Tabla 1. Parámetros cinéticos para la biosorción de AM sobre la BCT.

\begin{tabular}{ccccccccccc}
\hline \multirow{2}{*}{$\begin{array}{c}\mathrm{C}_{\mathrm{o}} \\
\mathrm{mg} / \mathrm{L}\end{array}$} & \multicolumn{1}{c}{ Pseudo primer orden } & \multicolumn{4}{c}{ pseudo segundo orden } & \multicolumn{3}{c}{ difusión intrapartícula } \\
\hline 50 & 0,12 & 43,33 & 0,9235 & 24,75 & 18,35 & 0,03 & 0,9997 & 3,05 & 74,67 & 0,9251 \\
100 & 0,15 & 22,53 & 0,8329 & 48,78 & 47,39 & 0,02 & 0,9999 & 2,57 & 34,90 & 0,8031 \\
200 & 0,24 & 36,97 & 0,9436 & 93,46 & 78,74 & 0,01 & 0,9999 & 0,99 & 19,16 & 0,9831 \\
\hline
\end{tabular}

\section{Isotermas de adsorción:}

Una isoterma de adsorción describe la dependencia entre el contaminante adsorbido y el contaminante en fase acuosa en condiciones de equilibrio y temperatura constante ${ }^{17}$. La figura 5 muestra las isotermas de biosorción a diferentes valores de $\mathrm{pH}$, la forma cóncava de las curvas de las isotermas indican la alta afinidad del adsorbato por el biosorbente, esta se asemeja a la isoterma del tipo L, según la clasificación de Giles et al. ${ }^{18}$ lo que sugiere una saturación progresiva del sólido sin alcanzar una capacidad límite, los datos experimentales fueron evaluados usando los modelos de isotermas de Langmuir y Freundlich, cuyas formas linealizadas se expresan en las ecuaciones 7 y 8 , respectivamente $^{5}$ :

$\frac{C_{e}}{q_{e}}=\frac{1}{q_{m a ́ x} b}+\frac{C_{e}}{q_{m a ́ x}}$

$\ln q_{e}=\ln K_{F}+\frac{1}{n} \ln C_{e}$

Donde: $\mathrm{q}_{\mathrm{e}}$ es la capacidad de biosorción en el equilibrio $(\mathrm{mg} / \mathrm{g})$; Ce es la concentración de equilibrio del $\mathrm{AM}$ en la fase líquida $(\mathrm{mg} / \mathrm{L}), \mathrm{q}_{\max } \mathrm{y}$ b son las constante de Langmuir, relacionadas con la máxima capacidad de biosorción para una monocapa completa $(\mathrm{mg} / \mathrm{g})$ y la afinidad entre el sorbente y el sorbato $(\mathrm{L} / \mathrm{mg})$, respectivamente, $K_{F}$ es la constante de equilibrio $(\mathrm{L} / \mathrm{g})$ y n es una constante relacionada con la afinidad entre el sorbente y el sorbato.

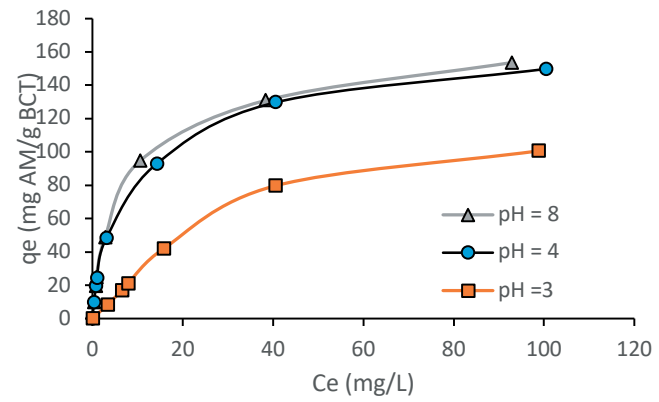

Figura 7. Isotermas del equilibrio de la biosorción de AM sobre la BCT, masa BCT/ volumen $=4 \mathrm{~g} / \mathrm{L}, \mathrm{T}=20 \mathrm{oC}, \mathrm{t}=1 \mathrm{~h}$. 
Las constantes de Langmuir (qmax y b) y Freundlich (KF y n) se calcularon a partir de la pendiente y la intersección de los gráficos lineales Ce/qe versus Ce (figura 8A) y log qe versus log Ce (figura 8B). Los parámetros de los modelos y los coeficientes de correlación se presentan en la tabla 2. En el caso del modelo de isoterma de Langmuir, se observó una muy buena correlación con los datos de biosorción basados en los coeficientes $\mathrm{R}^{2}$, los cuales son mayores a 0,96. La isoterma de Langmuir se basa en la suposición de que los sitios activos del biosorbente son homogéneos, este resultado sugiere la cobertura en monocapa de las moléculas del AM en la superficie de la BCT. El valor $1 / \mathrm{n}(0<1 / \mathrm{n}<1)$ obtenido de la isoterma de Freundlich indica una buena capacidad de absorción del AM sobre la BCT, el factor de separación RL se calculó para las tres isotermas y todos los valores se obtuvieron entre 0,$11 ; 0,02 ; 0,01$ lo que indica una biosorción favorable del AM sobre la BCT. Las capacidades máximas de biosorción suponiendo una biosorción en monocapa son 156,25; 158,73 y $156,25 \mathrm{mg} / \mathrm{g}$ a $\mathrm{pH} 3,4$ y 8 , respectivamente.
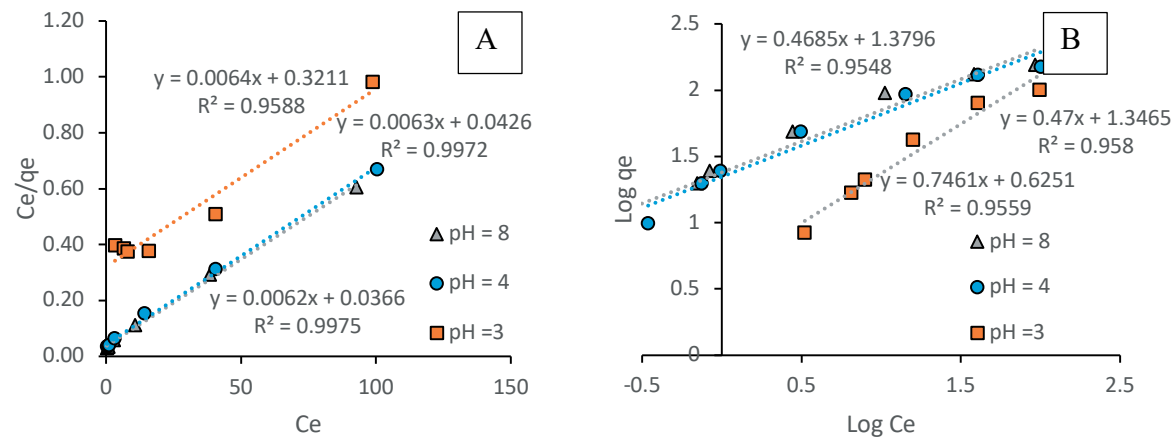

Figura 8. Estudio del equilibrio de la biosorción del AM A) Isoterma de Langmuir B) Isoterma de Freundlich

Tabla 2. Parámetros cinéticos para la biosorción de AM sobre la BCT.

\begin{tabular}{cccccccc}
\hline & \multicolumn{3}{c}{ Langmuir } & \multicolumn{4}{c}{ Freundlich } \\
\hline $\mathrm{pH}$ & $\mathrm{q}_{\max }(\mathrm{mg} / \mathrm{g})$ & $\mathrm{b}(\mathrm{L} / \mathrm{mg})$ & $\mathrm{R}_{\mathrm{L}}$ & $\mathrm{R}^{2}$ & $1 / \mathrm{n}$ & $\mathrm{K}_{\mathrm{F}}(\mathrm{L} / \mathrm{g})$ & $\mathrm{R}^{2}$ \\
\hline 3 & 156,25 & 0,02 & 0,11 & 0,9588 & 0,75 & 4,22 & 0,9559 \\
4 & 158,73 & 0,15 & 0,02 & 0,9972 & 0,47 & 22,21 & 0,9580 \\
8 & 156,25 & 0,26 & 0,01 & 0,9931 & 0,29 & 38,84 & 0,6791 \\
\hline
\end{tabular}

En comparación con otros estudios de biosorción del colorante AM reportados en la literatura (tabla 3), los altos valores de qmax demuestran que el material biosorbente BCT tiene un gran potencial para la eliminación del AM del medio acuoso. 
Tabla 3. Comparación de biosorción de AM con otros materiales biosorbentes.

\begin{tabular}{lll}
\hline Material biosorbente & $\mathrm{q}_{\max }(\mathrm{mg} / \mathrm{L})$ & Referencia \\
\hline Residuos de poda del árbol de olivo & 129,87 & Anastopoulus et al. ${ }^{19}$ \\
Madera de papaya & 32,25 & Rangabhashiyam et al. ${ }^{20}$ \\
Conchas de almejas & 1,09 & Elwakeel et al. ${ }^{15}$ \\
Residuos de pino & 129,87 & Yagub et al. ${ }^{21}$ \\
Alga gracilaria corticata & 95,41 & Vijayaraghavan et al. ${ }^{7}$ \\
Cladodios de tuna (Opuntia ficus indica) & 158,73 & Este estudio \\
\hline
\end{tabular}

\section{Influencia del pH:}

La eficiencia de la biosorción depende del $\mathrm{pH}$ de la solución, debido a que un cambio en el $\mathrm{pH}$ conduce a la variación en el grado de ionización de la molécula adsorbida y las propiedades superficiales del adsorbente. La figura 9 muestra el efecto del $\mathrm{pH}$ de la solución inicial sobre la capacidad de biosorción del colorante AM (qe) se observa que la cantidad adsorbida aumenta con el incremento del $\mathrm{pH}$, se encontró que la cantidad de colorante adsorbido aumentó de 24,87 mg/g a 99,16 mg/g debido al cambio en el pH de 2,0 a 8 para una concentración de colorante inicial de $200 \mathrm{mg} / \mathrm{L}$, el mismo comportamiento muestran las curvas a concentraciones iniciales menores $(50$ y $100 \mathrm{mg} / \mathrm{L})$. Es evidente que se logró mayores capacidades de remoción a un valor de $\mathrm{pH}$ 8. El valor del punto de carga cero $\left(\mathrm{pH}_{\mathrm{PCC}}\right)$ puede proporcionar información sobre la carga superficial del biosorbente (figura 3), cuando el $\mathrm{pH}>\mathrm{pH}_{\mathrm{PCC}}$, la superficie de la BCT se carga negativamente por lo que podría adsorber eficazmente el colorante catiónico AM por atracción electrostática traduciéndose en el incremento de la capacidad de biosorción ${ }^{15}$, contrariamente a $\mathrm{pH}<\mathrm{pH}_{\mathrm{PCC}}$, la superficie da la BCT se carga positivamente disminuyendo la capacidad de biosorción por repulsión electrostática ${ }^{2}$, por otro lado, la alta capacidad de eliminación del AM a pH alto también se debería a la presencia de menos $\mathrm{H}^{+}$que compiten por los sitios de sorción en la biomasa. El pH bajo conduce a un aumento en la concentración de iones $\mathrm{H}^{+}$en el sistema, y la superficie de la $\mathrm{BCT}$ adquiere carga positiva por protonación de grupos carboxilicos y amino de la superficie lo que conlleva a la disminución de la capacidad de adsorción del colorante catiónico $\mathrm{AM}^{17}$.

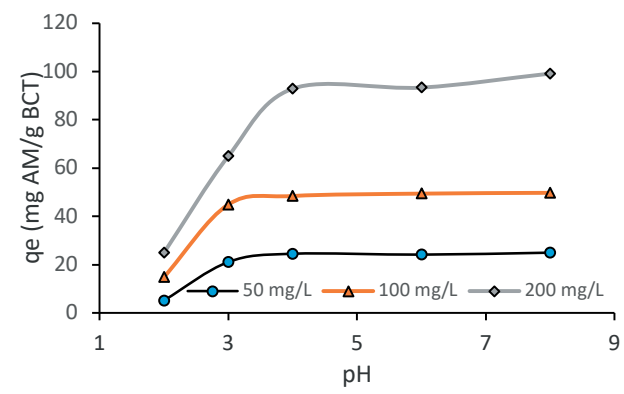

Figura 8. Influencia del $\mathrm{pH}$ sobre la capacidad de biosorción, dosis $\mathrm{BCT} / \mathrm{volumen}=$ $4 \mathrm{~g} / \mathrm{L}, \mathrm{T}=20 \mathrm{oC}, \mathrm{t}=120 \mathrm{~min}$. 


\section{Desorción:}

Para estudiar el potencial de reutilización del biosorbente se hicieron intentos para desorber el AM biosorbido en la BCT, para ello se empleó una solución de $\mathrm{HCl} \mathrm{0,01} \mathrm{M} \mathrm{como} \mathrm{agente}$ de desorción, con un tiempo de contacto de $120 \mathrm{~min}$. El rendimiento fue de 80,2 \%. Bajo condiciones ácidas fuertes, aumenta el número de sitios con carga positiva. Estos sitios con carga positiva en la superficie biosorbente favorecerían la desorción de los cationes del AM debido a la repulsión electrostática ${ }^{7}$.

\section{CONCLUSIONES}

Se investigó la remoción del AM de una solución acuosa uso la biomasa de los cladodios de la tuna (BCT) como material biosorbente. La concentración del biosorbente, el pH inicial de la solución, el tiempo de contacto jugaron un papel importante sobre la remoción del AM. Los resultados revelaron que el modelo cinético de pseudo-segundo orden se ajusta muy bien a la cinética de biosorción del AM sobre la BCT. Los datos de equilibrio de biosorción del AM coincidieron bien con el modelo de isoterma de Langmuir con una capacidad de adsorción de monocapa de 156,25 mg/g. El mecanismo de la biosorción se daría por un proceso de quimisorción e implicaría la adsorción sobre la superficie de la BCT y la difusión intrapartícula en los poros de la biomasa. Debido a que los cladodios de la tuna son un recurso natural, abundante, renovable, simple y ambientalmente amigable tiene un gran potencial para ser usado como biosorbente efectivo para la eliminación del colorante azul de metileno de los efluentes de aguas residuales.

\section{AGRADECIMIENTO}

Las autoras agradecen al Dr. Clemente Luyo de la Universidad Nacional Ingeniería por los análisis SEM/EDX realizados. 


\section{REFERENCIAS BIBLIOGRÁFICAS}

1. Alver E, Ülkü A, Brouers F. Methylene blue adsorption on magnetic alginate/rice husk biocomposite. Int J Biol Macromol. 2020; 154: 104-113.

2. Albadarin AB, Solomon S, Abou M, Walker G. Efficient removal of anionic and cationic dyes from aqueous systems using spent Yerba Mate "Ilex paraguariensis". J Taiwan Inst Chem Eng. 2018: 82: 144-155.

3. Afshariani F, Roosta A. Experimental study and mathematical modeling of biosorption of methylene blue from aqueous solution in a packed bed of microalgae Scenedesmus. J Clean Prod. 2019; 225: 133-142.

4. Han Q, Wang J, Goodman B, Xie J, Liu Z. High adsorption of methylene blue by activated carbon prepared from phosphoric acid treated eucalyptus residue. Powder Technol. 2020; 366: 239-248.

5. Moghazy R, Labena A, Husien Sh. Eco-friendly complementary biosorption process of methylene blue using micro-sized dried biosorbents of two macro-algal species (Ulva fasciata and Sargassum dentifolium): Full factorial design, equilibrium, and kinetic studies. Int J Biol Macromol. 2020; 134: 330-343.

6. Yagub MT, Kanti T, Afroze S, Ang HM. Dye and its removal from aqueous solution by adsorption: A review. Adv Colloid Interface Sci. 2014; 209: 172-184.

7. Vijayaraghavan J, Bhagavathi Pushpa T, Sardhar Basha SJ, Jegan J. Isotherm, kinetics and mechanistic studies of methylene blue biosorption onto red seaweed Gracilaria corticata, Desalination Water Treat. 2015; 1-9. doi: 10.1080/19443994.2015.1060174

8. León-Martínez F, Méndez-Lagunas L, Rodríguez-Ramírez J. Spray drying of nopal mucilage (Opuntia ficus-indica): Effects on powder properties and characterization. Carbohydr Polym. 2010; 81: 864-870.

9. Barka N, Abdennouri M, El Makhfouk M, Qourzal S. Biosorption characteristics of cadmium and lead onto eco-friendly dried cactus (Opuntia ficus indica) cladodes. J Environ Chem Eng. 2013; 1: 144-149.

10. Aygün A, Yenisoy -Karakaş S, Dumana I. Production of granular activated carbon from fruit stones and nutshells and evaluation of their physical, chemical and adsorption properties. Micropor Mesopor Mat. 2003; 6: 189-195.

11. Daneshvar E, Vazirzadeh A, Niazi A, Sillanpä M, Bhatnagar A. A comparative study of methylene blue biosorption using different modified brown, red and green macroalgae - Effect of pretreatment, Chem Eng J. 2017; 307 (1): 435-446.

12. Morosanu I, Teodosiu C, Paduraru C, Ibanescu D, Tofan L. Biosorption of lead ions from aqueous effluents by rapeseed biomass. N Biotechnol. 2017; 39: 110-124.

13. Nharingo T, Moyo M. Application of Opuntia ficus-indica in bioremediation of wastewaters. A critical review. J Environ Manag. 2016; 166: 55- 72.

14. Deniz F, Tezel E. A low-cost and eco-friendly biosorbent material for effective synthetic dye removal from aquatic environment: characterization, optimization, kinetic, isotherm and thermodynamic studies. Int J Phytoremediation. 2019; 22: 353-362.

15. Elwakeel KZ, Elgarahy AM, Mohamma SH. Use of beach bivalve shells located at Port Said coast (Egypt) as a green approach for methylene blue removal. J Environ Chem Eng. 2017; 5(1): 578-587. 
16. Nayak $\mathrm{AK}, \mathrm{Pa} \mathrm{A}$. Green and efficient biosorptive removal of methylene blue by Abelmoschus esculentus seed: Process optimization and multi-variate Modeling. J Environ Manag. 2017; 200: 145-159.

17. Afroze S, Kanti T, Anga M, Nishioka H. Adsorption of methylene blue dye from aqueous solution by novel biomass Eucalyptus sheathiana bark: equilibrium, kinetics, thermodynamics and Mechanism, Desalination Water Treat. 2016; 57(13): 5858-5878.

18. Giles C, Smith D, Huitson A. A general treatment and classification of the solute adsorption isotherm. I. Theoretical. J Colloid Interface Sci. 1974; 47(3): 755-765.

19. Anastopoulos I, Margiotoudis I, Massas I. The use of olive tree pruning waste compost to sequestrate methylene blue dye from aqueous solution. Int J Phytoremediation, 2018; 20(8): 831-838.

20. Rangabhashiyam S, Lata S, Balasubramanian P. Biosorption characteristics of methylene blue and malachite green from simulated wastewater onto Carica papaya wood biosorbent. Surf Interfaces. 2018; 10:197-215.

21. Yagub MT, Kanti Sen T, Ang M. Removal of cationic dye methylene blue (MB) from aqueous solution by ground raw and base modified pine cone powder. Environ Earth Sci. 2014; 71: 1507-1519. 